\title{
A VLASOV EQUATION WITH DIRAC POTENTIAL USED IN FUSION PLASMAS.
}

\author{
CLAUDE BARDOS AND ANNE NOURI
}

\begin{abstract}
Well-posedness of the Cauchy problem is analyzed for a singular Vlasov equation governing the evolution of the ionic distribution function of a quasineutral fusion plasma. The Penrose criterium is adapted to the linearized problem around a time and space homogeneous distribution function showing (due to the singularity) more drastic differences between stable and unstable situations. This pathology appears on the full non linear problem, well-posed locally in time with analytic initial data, but generally ill-posed in the Hadamard sense. Eventually with a very different class of solutions, mono-kinetic, which constrains the structure of the density distribution, the problem becomes locally in time well-posed.
\end{abstract}

\section{INTRODUCTION}

Instead of the standard Vlasov-Poisson equation for negatively charged particles in a fixed ionic background, or as denoted below VPE:

$$
\begin{aligned}
& \frac{\partial f}{\partial t}+v \cdot \nabla_{x} f-E(x) \cdot \nabla_{v} f=0, \quad t>0, \quad(x, v) \in \mathbb{R}_{x}^{D} \times \mathbb{R}_{v}^{D}, \\
& E=-\nabla_{x} \phi, \quad \Delta_{x} \phi=\rho(t, x)-1=\int_{\mathbb{R}_{v}^{D}} f\left(t, x, v^{\prime}\right) d v^{\prime}-1,
\end{aligned}
$$

where the electric field is computed according to the Poisson law, we consider in this contribution the following problem:

$$
\frac{\partial f}{\partial t}+v \frac{\partial f}{\partial x}-\left(\frac{\partial}{\partial x} \int f(t, x, u) d u\right) \frac{\partial f}{\partial v}=0, \quad t>0, \quad(x, v) \in \mathbb{R}_{x} \times \mathbb{R}_{v}
$$

which is a one dimensional version of (1.1) where, in the computation of the electric field, the Poisson Kernel which gives the inverse of the Laplacian, is replaced by the Dirac mass. Hence to avoid confusion with the Vlasov Dirac equation describing the coupling of a density of ions with a Dirac equation, we call it the Vlasov equation with Dirac Potential or in short VDPE.

As explained in Section 2, it turns out that this equation is used in plasma physics and in particular in nuclear fusion.

From the mathematical point of view the first observation is that the VDPE is more singular: the mapping $\rho \mapsto E$ being now an operator of degree 1 instead of being an operator of degree -1 as it was the case for the VPE.

This explains the pathologies of the problem. Following what is already classical for the VPE, the first step is to study the linearized (near a space independent distribution $G(v)$ ) version of the VDPE. The Penrose criterium can be adapted. For the VPE it gave a necessary and sufficient condition for the existence of smooth but exponentially (with time) growing solutions. Here it makes a more drastic difference between situations where the problem is badly posed, except for short time and with analytic initial data (as it is for instance the case for the Kelvin Helmholtz equation) and situations where it can be described by semigroup theory. This will be the object of Section 3.

This explains also why a general existence theorem can be obtained only locally in time in the class of analytic initial data (cf. [15]) and why one can construct with the unstable modes of the

\footnotetext{
${ }^{1} 2010$ Mathematics Subject Classification: 82B40, 82C26.

${ }^{2}$ Key words: Vlasov equation, well-posed Cauchy problem, ill-posed Cauchy problem, O.Penrose criterium.
} 
linearized equations solutions of the non linear problem of arbitrary high Sobolev regularity and arbitrary short time of existence as explained in Section 4.

Eventually as it has been observed already at least in (1969) in [7] for mono-kinetic densities of the form $f(t, x, v)=\rho(t, x) \delta(v-u(t, x))$, the VDPE turns out to be equivalent to a system of isentropic gas dynamics for $(\rho, u)$, hence well-posed for initial data in Sobolev spaces. This is recalled in Section 6 mostly to underline the fact that the notion of well-posedness versus illposedness is also functional-space dependent and that a problem badly posed in a general setting may turn out to be tamed in a situation where singular perturbations are not allowed to appear.

This contribution concerns a problem of Mathematical Physics and it is for us a pleasure and an honor to dedicate it to Peter Constantin as a token of friendship and recognition of the essential role that he played over the years in this field.

\section{Pertinence of the VDPE in the modeling of plasma fusion.}

In this section, we recall the main lines of the derivation of a model used for studying fusion plasmas, performed in [10]. The evolution of the ions in the core of the plasma is well described by the Vlasov equation,

$$
\frac{\partial f}{\partial t}+v \cdot \nabla_{x} f+\frac{Z e}{m_{i}}\left(-\nabla_{x} \Phi+v \wedge B\right) \cdot \nabla_{v} f=0
$$

where $f$ is the ionic distribution function depending on time, position (in the domain $\Omega$ of the plasma) and velocity (in $\mathbb{R}^{3}$ ), $Z e$ and $m_{i}$ are the ion charge and mass respectively. A priori, the electric potential $\Phi$ and the magnetic field $B$ are governed by the Maxwell equations. But a finite Larmor radius approximation is derived in the limit of a large and uniform external magnetic field. This leads to the following equation for the ionic distribution function $\bar{f}$ in gyrocoordinates,

$$
\frac{\partial \bar{f}}{\partial t}+v_{\|} \frac{\partial \bar{f}}{\partial x_{\|}}-J_{\rho_{L}}^{0}\left(\frac{\partial \Phi}{\partial x_{\|}}\right) \frac{\partial \bar{f}}{\partial v_{\|}}-\left(J_{\rho_{L}}^{0} \nabla_{x_{\perp}} \Phi\right)^{\perp} \cdot \nabla_{x} \bar{f}=0 .
$$

Here, the index $\|($ resp. $\perp$ ) refers to the direction parallel (resp. orthogonal) to the external magnetic field. For any vector $u=\left(u_{i}\right) \in \mathbb{R}^{3}, u^{\perp}$ denotes the vector $\left(u_{2},-u_{1}, 0\right)$. The operator $J_{\rho_{L}}^{0}$ is a Bessel operator performing averages on circles of Larmor radius $\rho_{L}$ in planes orthogonal to the magnetic field. We shall not use it in this paper, so we do not enter into more details about it. The electrons moving quite more quickly than the ions are assumed to be adiabatic, i.e. their density $n_{e}$ is given in terms of the electric potential $\Phi$ by

$$
n_{e}=n_{0} e^{\frac{e}{T_{e}}(\Phi-<\Phi>)} .
$$

Here, $e$ (resp. $T_{e}$ ) is the electronic charge (resp. temperature), and $\langle\Phi\rangle$ is the average of the potential on a magnetic field line. The system is assumed close to equilibrium, i.e. the departure from a constant electric potential is small. Equation (2.3) simplifies into

$$
n_{e}=n_{0}\left(1+\frac{e}{T_{e}}(\Phi-<\Phi>)\right) .
$$

Denote by

$$
\rho(t, x)=\int f(t, x, v) d v
$$

the ionic density. Due to the electroneutrality of the plasma,

$$
\rho(t, x)=n_{e}(t, x), \quad t>0, \quad x \in \Omega,
$$

the electric field $-\nabla_{x} \Phi$ appearing in (2.2) is proportional to $-\nabla_{x} \rho$. The operator $J_{\rho_{L}}^{0}$ induces some regularity in the orthogonal direction, but none in the parallel direction. The two-dimensional dynamics in the direction perpendicular to the magnetic field is studied in [14]. In order to analyze the difficulty coming from the highly singular term $J_{\rho_{L}}^{0}\left(\frac{\partial \Phi}{\partial x_{\|}}\right) \frac{\partial \bar{f}}{\partial v_{\|}}$, we restrict to a one-dimensional spatial setting, e.g. by considering ionic distribution functions written in the form

$$
f(t, x, v)=f_{\|}\left(t, x_{\|}, v_{\|}\right) f_{\perp}\left(\left|v_{\perp}\right|\right),
$$


with

$$
\int_{0}^{+\infty} f_{\perp}\left(\left|v_{\perp}\right|\right) 2 \pi\left|v_{\perp}\right| d\left|v_{\perp}\right|=1
$$

Then the term $f_{\perp}$ has no incidence in the Vlasov equation and can be factorized. The equation that $f_{\|}$should solve is

$$
\frac{\partial f_{\|}}{\partial t}+v_{\|} \frac{\partial f_{\|}}{\partial x_{\|}}-\left(\frac{\partial}{\partial x_{\|}} \int f_{\|}\left(t, x_{\|}, u\right) d u\right) \frac{\partial f_{\|}}{\partial v_{\|}}=0, \quad t>0, \quad\left(x_{\|}, v_{\|}\right) \in \mathbb{R}^{2} .
$$

From now on, denote by $f$ (resp. $x, v), f_{\|}\left(\operatorname{resp} . x_{\|}, v_{\|}\right)$for the sake of simplicity.

\section{Properties of the Linearized Equation}

First it is appropriate to compare the properties of the linearized VDPE with the linearized VPE. For a fluctuation near an homogenous density profile:

$$
g(0, x, v)=f_{i}(x, v)-G(v), \quad(x, v) \in \mathbb{R}^{2},
$$

with $A_{D}$ denoting the advection operator:

$$
g \mapsto A_{D}(g)=v \cdot \partial_{x} g,
$$

both are written:

$$
\frac{\partial g}{\partial t}+A_{D}(g)-E(g) G^{\prime}(v)=0, \quad t>0, \quad(x, v) \in \mathbb{R}^{2}
$$

with

$$
\begin{aligned}
& \text { for } \operatorname{VPE} \quad E(g)=E(g)_{V P E}=-\partial_{x} \phi \text { with } \partial_{x}^{2} \phi=\rho+\rho_{G}-1=\int_{\mathbb{R}_{v}} g\left(\cdot, \cdot, v^{\prime}\right) d v^{\prime}+\rho_{G}-1, \\
& \text { for } \operatorname{VDPE} \quad E(g)=E_{V D P E}(g)=\partial_{x} \rho=\partial_{x} \int_{\mathbb{R}_{v}} g\left(\cdot, \cdot, v^{\prime}\right) d v^{\prime} .
\end{aligned}
$$

As in the case of the linearized VPE the coefficients of the linearized VDPE equation do not depend on $x$. Therefore in spite of the pathologies of this equation (to be studied below) one has the following uniqueness theorem:

Theorem 3.1. Assume that

$$
v \mapsto G^{\prime}(v) \in L^{1}(\mathbb{R}) .
$$

Then any $g(t, x, v) \in L^{1}\left(0, T ; L^{2}\left(\mathbb{R} ; L^{1}(\mathbb{R})\right)\right)$ solution for $t \in[0, T]$ of the linearized VDPE

$$
\partial_{t} g+v \cdot \partial_{x} g-\partial_{x}\left(\int_{\mathbb{R}_{v}} g\left(t, x, v^{\prime}\right) d v^{\prime}\right) G^{\prime}(v)=0
$$

with initial condition

$$
g(0, x, v)=0, \quad(x, v) \in \mathbb{R}^{2},
$$

is identically zero.

Proof. With the Fourier transform $x \mapsto k, \hat{g}(t, k, v)$ satisfies the equation

$$
\partial_{t} \hat{g}+i k v \hat{g}-i k \hat{\rho}(t, k) G^{\prime}(v)=0 \quad \text { with } \hat{\rho}(t, k)=\int_{\mathbb{R}_{v}} \hat{g}\left(t, x, v^{\prime}\right) d v^{\prime},
$$

together with

$$
\hat{g}(0, k, v)=0,(k, v) \in \mathbb{R}^{2}
$$

Hence,

$$
\hat{g}(t, k, v)=i k G^{\prime}(v) \int_{0}^{t} \hat{\rho}(s, k) e^{i k v(s-t)} d s
$$


and

$$
\hat{\rho}(t, k)=i k \int_{0}^{t} \hat{\rho}(s, k) \int_{\mathbb{R}_{v}} G^{\prime}(v) e^{i k v(s-t)} d v d s .
$$

Let $R>0$ be given. Then

$$
\sup _{|k|<R}|\hat{\rho}(t, k)| \leq R\left\|G^{\prime}\right\|_{L^{1}} \int_{0}^{t} \sup _{|k|<R}|\hat{\rho}(s, k)| d s, \text { and } \hat{\rho}(0, k)=0,|k|<R \text {. }
$$

Hence

$$
\hat{\rho}(t, k)=0, \quad t \in[0, T], \quad|k|<R .
$$

Since this holds for any positive $R$, the function $\hat{\rho}$ is identically zero. Then $g$ satisfies

$$
\begin{array}{rr}
\frac{\partial g}{\partial t}+v \frac{\partial g}{\partial x}=0, \quad t \in(0, T), & (x, v) \in \mathbb{R}^{2}, \\
g(0, x, v)=0, & (x, v) \in \mathbb{R}^{2},
\end{array}
$$

so that $g$ is identically zero.

Remark 1. The previous uniqueness theorem result can be extended to any distribution $(t, x) \mapsto$ $g(t, x, v)$ defined on $(0, T) \times R_{x}$ with values in $L^{1}\left(\mathbb{R}_{v}\right)$, solution of (3.5) and identically zero for $t=0$. Indeed, introduce with $R>0$ (as above) the Fourier regularizing operator

$$
P_{R} g(t, x, v)=\int_{-R}^{R} e^{i k x} \hat{g}(t, k, v) d k .
$$

Then observe that $P_{R} g$ belongs to $L^{1}\left(0, T ; L^{2}\left(\mathbb{R} ; L^{1}(\mathbb{R})\right)\right)$ and is a solution of (3.5). The result follows from the above theorem.

Restricted to any standard space $\mathcal{B}$ (in particular to $\mathcal{B}=L^{2}\left(\mathbb{R}_{x} ; L^{1}\left(\mathbb{R}_{v}\right)\right)$ ) with domain

$$
D\left(A_{D}\right)=\left\{g \in \mathcal{B} \quad \text { such that } \quad A_{D} g \in \mathcal{B}\right\}
$$

$A_{D}$ is the generator of a continuous group of operator and $g \mapsto E_{V P E}(g)$ is an operator of degree -1 and therefore bounded in $\mathcal{B}$. Hence $-\left(A_{D}+E_{V P E}\right)$ is the generator of a strongly continuous group of operators. The spectrum $\sigma\left(-\left(A_{D}+E_{V P E}\right)\right)$ is contained in a slab $-a \leq \Re \lambda \leq a$. With initial data in $\mathcal{B}$, the initial value problem (with data given for $t=0$ ) is well posed both for positive and negative times. The essential remaining issue is the stability or the asymptotic behavior of the solution for $t \rightarrow \pm \infty$.

On the other hand as will be shown below, since the operator $g \mapsto E_{V D P E}(g)$ is unbounded (of degree -1) this is no more the case for the VDPE Cauchy problem. And the fact that the problem will be well posed or not well posed will turn out to be one of the essential issues.

Since the coefficients of the above equations are $x$ independent one can use the $x \mapsto k$ Fourier transform and ( this has been done since Vlasov himself [22]) the modal analysis (cf also [5] for a complete functional analysis based on modal analysis and Fourier transform).

Looking for modes i.e. solutions of the form :

$$
e(t, x, v, k)=e^{i(k x-\omega(k) k t)} a(v, k),
$$

leads for VPE and VDPE, with $\hat{\rho}(k)=\int_{\mathbb{R}_{v}} a(v, k) d v$, to the following equations

$$
\begin{array}{rlr}
(-i \omega k+i k v) a(v, k)-i \frac{k}{k^{2}} G^{\prime}(v) \hat{\rho}(k) & =0 & \text { for VPE } \\
\text { and } \quad(-i \omega k+i k v) a(v, k)-i k G^{\prime}(v) \hat{\rho}(k) & =0 & \text { for VDPE. }
\end{array}
$$

These equations are solved, with the introduction of the dispersion relation, by the formulas:

$$
k^{2}-\int_{\mathbb{R}_{v}} \frac{G^{\prime}(v)}{v-\omega} d v=0, \quad a(v, k)=\frac{G^{\prime}(v)}{v-\omega} \frac{\hat{\rho}(k)}{k^{2}},
$$


for the VPE and by

$$
1-\int_{\mathbb{R}_{v}} \frac{G^{\prime}(v)}{v-\omega} d v=0, \quad a(v, k)=\frac{G^{\prime}(v)}{v-\omega} \hat{\rho}(k),
$$

for the VDPE.

Now as observed by Penrose [21], unstable modes in VPE correspond to solutions $\omega(k)$ of $(3.11)$ with $\Im \omega>0$. With such modes $\omega(k)$ one can reconstruct by Fourier synthesis (cf [5] for details) solutions of the form:

$$
g_{V P E}(t, x, v)=\int_{\mathbb{R}_{k}} e^{i(k x-\omega(k) k t)} \frac{G^{\prime}(v)}{v-\omega} \frac{\hat{\rho}(k)}{k^{2}} d k,
$$

which will be exponentially growing in time.

Therefore (still following [21]), denoting by

$$
Z\left(\Im_{+}\right)=\left\{\int_{\mathbb{R}_{v}} \frac{G^{\prime}(v)}{v-\omega} d v \text { with } \Im \omega>0\right\},
$$

the image of the upper half plane $\Im_{+}$under the map

$$
\omega \mapsto Z(\omega)=\int_{\mathbb{R}_{v}} \frac{G^{\prime}(v)}{v-\omega} d v
$$

such an $\omega(k)$ exists if and only if $Z\left(\Im_{+}\right)$contains a real and positive number $k^{2}$.

Now $Z\left(\Im_{+}\right)$is a bounded set and its boundary is the curve:

$$
\mathcal{C}_{+}: v^{*} \in \mathbb{R} \mapsto Z\left(v^{*}+i 0\right)=\int_{\mathbb{R}_{v}} \frac{G^{\prime}(v)}{v-\left(v^{*}+i 0\right)} d v .
$$

$\mathcal{C}_{+}$is a direct bounded and continuous curve starting and ending at the origin:

$$
\lim _{\left|v^{*}\right| \rightarrow \pm \infty} Z\left(v^{*}+i 0\right)=0 .
$$

Since this curve is bounded, one then observes the existence of some $M$ such that for $|k|>M$, the equation (3.11) has no solution. This observation is in agreement with the fact that the linearized VPE is always well-posed but may be with exponentially growing modes whenever $Z\left(\Im_{+}\right)$contains real positive numbers.

Eventually the difference between (3.11) and (3.12) comes from the fact that this later equation is independent of $k$. If it has a solution $\omega^{*}$ then (cf for instance Theorem 3.2 below) the formal solution

$$
g_{V D P E}(t, x, v)=\int_{\mathbb{R}_{k}} e^{i k\left(x-\omega^{*} t\right)} \frac{G^{\prime}(v)}{v-\omega^{*}} \hat{\rho}(k) d k
$$

will be used to discuss the instabilities of the linearized and the non linear VDPE equation.

\subsection{Instabilities of the linearized problem.}

Theorem 3.2. For the stationary profile $v \mapsto G(v) \in L^{1}(\mathbb{R}) \cap C^{2}(\mathbb{R})$, assume the existence of a point $v^{*} \in \mathbb{R}$ with the following properties:

$$
G^{\prime}\left(v^{*}\right)=0 \quad \text { and } \int_{\mathbb{R}_{v}} \frac{G^{\prime}(v)}{v-v^{*}} d v>1 .
$$

Then there exist a solution $\omega^{*}$ with $\Im \omega^{*}>0$ of the equation

$$
1-\int_{\mathbb{R}_{v}} \frac{G^{\prime}(v)}{v-\omega^{*}} d v=0,
$$

and for any $k \in \mathbb{R}$ an unstable mode of the form:

$$
e(t, x, v)=e^{i k\left(x-\omega^{*} t\right)} \frac{G^{\prime}(v)}{v-\omega^{*}} \hat{\rho}(k) .
$$


Remark 2. With $G^{\prime}\left(v^{*}\right)=0$, the integral

$$
\int_{\mathbb{R}_{v}} \frac{G^{\prime}(v)}{v-v^{*}} d v
$$

is well defined. With $\tilde{G}(v)=G(v)-G\left(v^{*}\right)$ it coincides (the integration by part being valid) with:

$$
\int_{\mathbb{R}_{v}} \frac{\tilde{G}(v)}{\left(v-v^{*}\right)^{2}} d v
$$

Proof of Theorem 3.2. The proof is a direct adaptation of [21]. 1 belongs to $Z\left(\Im_{+}\right)$if and only if there exists a point $v^{*}$ which satisfies the hypothesis (3.18). With the existence of an unstable mode, the fact that the linearized problem may be badly posed can be stated according to the

Corollary 3.3. With the hypothesis of Theorem 3.2, for any $T>0$ there is a function

$$
g_{i} \in \cap_{m \in \mathbf{N}} H^{m}\left(\mathbb{R} ; L^{1}(\mathbb{R})\right),
$$

such that the Cauchy problem with initial datum $g_{i}$ for the linearized problem around $G$ has for any $\tilde{T}<T$ and any $m \in \mathbf{N}^{*}$ a unique solution in $L^{\infty}\left(0, \tilde{T} ; H^{m}\left(\mathbb{R} ; L^{1}(\mathbb{R})\right)\right)$. Moreover,

$$
\lim _{t \rightarrow T^{-}}\|g(t, \cdot, \cdot)\|_{H^{m}\left(\mathbb{R} ; L^{2}(\mathbb{R})\right)}=+\infty .
$$

Proof. Assume that $\hat{\rho}(k)$ is for $|k|$ large equivalent to $\exp \left(-\Im \omega^{*} T|k|\right)$ and consider the function

$$
g(t, x, v)=\int_{\mathbb{R}_{k}} e^{i k\left(x-\omega^{*} t\right)} \frac{G^{\prime}(v)}{v-\omega^{*}} \hat{\rho}(k) d k,
$$

which is, for $0<t<T$, a smooth solution of the VDPE with initial data

$$
g_{i n}=g(0, x, v)=\int_{\mathbb{R}_{k}} e^{i k x} \frac{G^{\prime}(v)}{v-\omega^{*}} \hat{\rho}(k) d k .
$$

Hence by the uniqueness theorem it is the only solution and it blows up (even as a distribution) for $t \rightarrow T^{-}$.

Remark 3. Real valued instabilities can be deduced from Corollary 3.3.

Indeed, consider $\hat{\rho}$ as in the proof of Corollary 3.3, that is also real valued and even. Observe that both

$$
g_{1}(t, x, v)=\frac{G^{\prime}(v)}{v-i \Im \omega^{*}} \int e^{i k x+\Im \omega^{*} k t} \hat{\rho}(k) d k
$$

and

$$
g_{2}(t, x, v)=\frac{G^{\prime}(v)}{v+i \Im \omega^{*}} \int e^{i k x-\Im \omega^{*} k t} \hat{\rho}(k) d k=\frac{G^{\prime}(v)}{v+i \Im \omega^{*}} \int e^{-i k x+\Im \omega^{*} k t} \hat{\rho}(k) d k
$$

are solutions of (3.5). Therefore,

$$
\phi(t, x, v)=\frac{1}{2}\left(g_{1}+g_{2}\right)(t, x, v)=\frac{G^{\prime}(v)}{\left(\Im \omega^{*}\right)^{2}+v^{2}} \int e^{\Im \omega^{*} k t}\left(v \cos k x-\Im \omega^{*} \sin k x\right) \hat{\rho}(k) d k
$$

is a real valued solution of (3.5) with initial datum given by:

$$
\phi(0, x, v)=\frac{G^{\prime}(v)}{\left(\Im \omega^{*}\right)^{2}+v^{2}} \int\left(v \cos k x-\Im \omega^{*} \sin k x\right) \hat{\rho}(k) d k \in \cap_{m \in \mathbf{N}} H^{m}\left(\mathbb{R} ; L^{1}(\mathbb{R})\right) .
$$

Moreover,

$$
\begin{gathered}
\int|\hat{\phi}(t, k, v)| d v=|\hat{\rho}(k)| \int\left|G^{\prime}(v)\right|\left|\frac{e^{\Im \omega^{*} k t}}{v-i \Im \omega^{*}}-\frac{e^{-\Im \omega^{*} k t}}{v+i \Im \omega^{*}}\right| d v \\
=2|\hat{\rho}(k)| \int\left|G^{\prime}(v)\right|\left(\frac{s h^{2} \Im \omega^{*} k t}{\left(\Im \omega^{*}\right)^{2}+v^{2}}+\frac{v^{2}}{\left(\left(\Im \omega^{*}\right)^{2}+v^{2}\right)^{2}}\right)^{\frac{1}{2}} d v .
\end{gathered}
$$


Hence

$\|\phi(t, \cdot, \cdot)\|_{H^{m}\left(\mathbb{R} ; L^{1}(\mathbb{R})\right)}^{2}=4 \sum_{j=0}^{m} \int_{\mathbb{R}}|k|^{2 j}|\hat{\rho}(k)|^{2}\left(\int\left|G^{\prime}(v)\right|\left(\frac{s h^{2} \Im \omega^{*} k t}{\left(\Im \omega^{*}\right)^{2}+v^{2}}+\frac{v^{2}}{\left(\left(\Im \omega^{*}\right)^{2}+v^{2}\right)^{2}}\right)^{\frac{1}{2}} d v\right)^{2} d k$

is uniformly bounded on any interval $[0, \tilde{T}]$ with $\tilde{T}<T$. Eventually, $\|\Phi(t, \cdot, \cdot)\|_{H^{m}\left(\mathbb{R} ; L^{1}(\mathbb{R})\right)}$ tends to infinity when $t$ tends to $T^{-}$, since

$$
\|\phi(t, \cdot, \cdot)\|_{H^{m}\left(\mathbb{R} ; L^{1}(\mathbb{R})\right)}^{2} \geq c_{1}\left(\int \frac{\left|G^{\prime}(v)\right|}{\sqrt{\left(\Im \omega^{*}\right)^{2}+v^{2}}} d v\right)^{2} \sum_{j=0}^{m} \int_{\mathbb{R}}|k|^{2 j} \mid e^{-2 \Im \omega^{*}|k| T} s h^{2}\left(\Im \omega^{*} k t\right) d k,
$$

for some constant $c_{1}$.

3.2. Stability near a convenient profile. To underline the role of the bumps a stability theorem converse of the linearized instability theorem is proven again following the method of [21].

Denote by $C_{b}^{2}(\mathbb{R})$ the space of functions with second derivative continuous and bounded.

Introduce the space $\mathcal{B}$ of distributions $f(x, v)$ such that their Fourier transform in space, $\hat{f}(k, v)$, belongs to $L^{2}\left(\mathbb{R}_{k} ; L^{1}\left(\mathbb{R}_{v}\right)\right)$ with the natural norm:

$$
\|f\|_{\mathcal{B}}^{2}=\int_{\mathbb{R}_{k}}\left(\int_{\mathbb{R}_{v}}|\hat{f}(k, v)| d v\right)^{2} d k .
$$

Then define the operator $A$ by

$$
\begin{array}{r}
D(A)=\left\{g \in \mathcal{B} ; v \cdot \partial_{x} g-\partial_{x} \rho G^{\prime}(v) \in \mathcal{B}\right\}, \\
A g=-v \cdot \partial_{x} g+\partial_{x} \rho G^{\prime}(v) .
\end{array}
$$

In this setting one has the:

Proposition 3.1. Assume for the profile $v \mapsto G(v) \in C_{b}^{2}\left(\mathbb{R}_{v}\right) \cap L^{1}\left(\mathbb{R}_{v}\right)$ the following property,

$$
G^{\prime}(0)=0 \text { and } \quad G^{\prime}\left(v^{*}\right)=0 \Rightarrow \int_{\mathbb{R}_{v}} \frac{G^{\prime}(v)}{v-v *} d v<1
$$

Then the spectrum of $A$ is contained in the imaginary axis. Moreover, for $\lambda \in \mathbb{C}$ with $|\Re \lambda|>0$, the resolvent $(\lambda I-A)^{-1}$ is bounded in $\mathcal{B}$ according to the estimate:

$$
\left.\left\|(\lambda I-A)^{-1}\right\|_{\mathcal{B}} \leq\left(\frac{1}{|\Re \lambda|}+\frac{1}{\alpha}\left(1+\frac{|\Im \lambda|}{|\Re \lambda|}\right) \int\left|\frac{G^{\prime}(v)}{v}\right| d v\right)\right) .
$$

Proof of the proposition 3.1

With $h \in \mathcal{B}$ start from the resolvent equation in Fourier space:

$$
(\lambda+i k v) \hat{g}-i k \hat{\rho} G^{\prime}(v)=\hat{h}, \quad \hat{\rho}=\int_{\mathbb{R}_{v}} \hat{g}(v, k) d v,
$$

which, for $\Re \lambda \neq 0$ and $\omega=i \lambda / k$, is equivalent to

$$
\begin{aligned}
& \left(1-\int_{\mathbb{R}_{v}} \frac{G^{\prime}(v)}{v-\omega} d v\right) \hat{\rho}(k)=\int_{\mathbb{R}_{v}} \frac{\hat{h}(k, v)}{\lambda+i k v} d v, \\
& \hat{g}(k, v)=\frac{\hat{h}(k, v)}{\lambda+i k v}+\hat{\rho}(k) \frac{i k G^{\prime}(v)}{\lambda+i k v} .
\end{aligned}
$$

Following again [21], one introduces the sets $Z\left(\Im_{ \pm}\right)$images of the half planes

$$
\Im_{ \pm}=\{\omega \in \mathbb{C} \text { such that } \pm \Im \omega>0\},
$$

under the mapping

$$
\omega \mapsto Z(\omega)=\int \frac{G^{\prime}(v)}{v-\omega} d v
$$


As above $Z\left(\Im_{ \pm}\right)$are bounded subsets of $\mathbb{C}$ with boundary given by the curves

$$
C_{ \pm}: \gamma \mapsto \int \frac{G^{\prime}(v)}{v-\gamma \pm i 0} d v
$$

These two curves are bounded continuous with limit points $C_{ \pm}( \pm \infty)=0$. As in [21] the relation (3.29) implies that 1 does not belong to the sets $C_{ \pm}$. Since these curves are bounded and continuous it follows that;

$$
\forall \omega \in \mathbb{C} \backslash \mathbb{R}, \quad\left|1-\int_{\mathbb{R}_{v}} \frac{G^{\prime}(v)}{v-\omega} d v\right| \geq \operatorname{dist}\left(1, Z\left(\Im_{ \pm}\right)\right) \geq \operatorname{dist}\left(1, C_{ \pm}\right) \geq \alpha>0 .
$$

Coming back to the variable $\lambda=-i \omega k$ and denoting by

$$
R(\lambda, k)=1-\int \frac{i k G^{\prime}(v)}{\lambda+i k v} d v
$$

one has:

$$
|R(\lambda, k)| \geq \alpha .
$$

Therefore $\hat{g}(k, v)$ is uniquely given by the formula:

$$
\hat{g}(k, v)=\frac{\hat{h}(k, v)}{\lambda+i k v}+\frac{i k v \frac{G^{\prime}(v)}{v}}{\lambda+i k v} \frac{1}{R(\lambda, k)} \int \frac{\hat{h}(k, w)}{\lambda+i k w} d w .
$$

With the hypothesis $G^{\prime}(0)=0$, crude estimates like

$$
\begin{aligned}
& \int_{\mathbb{R}_{v}} \frac{|\hat{h}(k, v)|}{|\lambda+i k v|} d v \leq \frac{1}{|\Re \lambda|} \int_{\mathbb{R}_{v}}|\hat{h}(k, v)| d v, \\
& \int_{\mathbb{R}_{v}}\left|\frac{i k v \frac{G^{\prime}(v)}{v}}{\lambda+i k v}\right| d v \leq\left(1+\frac{|\Im \lambda|}{|\Re \lambda|}\right) \int_{\mathbb{R}_{v}}\left|\frac{G^{\prime}(v)}{v}\right| d v,
\end{aligned}
$$

eventually give:

$$
\int|\hat{g}(k, v)| d v \leq\left(\frac{1}{|\Re \lambda|}+\frac{1}{\alpha}\left(1+\frac{|\Im \lambda|}{|\Re \lambda|}\right)\left(\int\left|\frac{G^{\prime}(v)}{v}\right| d v\right) \int|\hat{h}(k, v)| d v .\right.
$$

This proves (3.30).

Corollary 3.4. Under the hypothesis of Theorem 3.1, the operator $A$ is the generator of a distribution group in the space $\mathcal{B}$ and for initial data in $D\left(A^{k}\right)$ (with $k$ large enough) the Cauchy problem (3.5)-(3.1) has a unique solution in $C^{1}\left(\mathbb{R}_{t}^{+} ; \mathcal{B}\right) \cap C^{0}\left(\mathbb{R}_{t}^{+} ; \mathcal{B}\right)$.

Proof. By Theorem 3.1, the operator $A$ satisfies the hypothesis of Theorem 1.6 of [3], which explicitly gives this corollary.

Remark 4. For $\lambda$ positive and real, one can estimate the right hand side of (3.37) using the formula

$$
\int\left|\frac{1}{\lambda+i k v}\right|^{2} d v=\frac{\pi}{\lambda|k|}
$$

and the Cauchy Schwartz inequality to obtain:

$$
\left\|(\lambda I-A)^{-1}\right\|_{\mathcal{B}} \leq \frac{C}{\lambda}
$$

with $C>1$. The estimate (3.38) with $\frac{C}{\lambda}$ replaced by $\frac{1}{\lambda-a}$ for $\lambda>$ a taken large enough would be enough to characterize A (with the Hille Yosida theorem) as the generator of a classical strongly continuous semi group of operators, for which the classical Cauchy problem is well posed with no loss of regularity. However we have not been able to introduce $\frac{1}{\lambda-a}$ instead of $\frac{C}{\lambda}$ in the right hand side of (3.38). This is due to presence of $i k$ in the singular term

$$
\frac{i k v \frac{G^{\prime}(v)}{v}}{\lambda+i k v} \frac{1}{R(\lambda, k)} \int \frac{\hat{g}(k, w)}{\lambda+i k w} d w .
$$


This is the reason why the notion of distribution semi groups has been introduced by several authors [19], [4] and systematically studied in [3]. It corresponds to a loss of regularity with respect to the initial datum.

Moreover, in the present case it can be illustrated by a direct computation which in Corollary 3.4 gives $k=4$. This is the object of the following

Theorem 3.5. Under the hypothesis of Proposition 3.1, for any $g_{i} \in D\left(A^{4}\right)$, the Cauchy problem

$$
\partial_{t} g+v \cdot \partial_{x} g-\partial_{x}\left(\int_{\mathbb{R}_{v}} g\left(t, x, v^{\prime}\right) d v^{\prime}\right) G^{\prime}(v)=0,\left(\text { or } \partial_{t} g=A g\right),
$$

with the initial condition

$$
g(0, x, v)=g_{i}(x, v), \quad(x, v) \in \mathbb{R}^{2},
$$

has a unique solution in $C^{1}\left(\mathbb{R}_{t}^{+} ; \mathcal{B}\right) \cap C^{0}\left(\mathbb{R}_{t}^{+} ; \mathcal{B}\right)$.

\section{Proof}

Reproducing the basic arguments of [3], define $g(t, x, v)$ by the inverse Laplace transform (with $\lambda=\gamma+i \sigma, \gamma=\Re \lambda>0)$ :

$$
g(t, x, v)=\frac{1}{2 i \pi} \lim _{\sigma \rightarrow \infty} \int_{\gamma-i \sigma}^{\gamma+i \sigma} e^{\lambda t}(\lambda I-A)^{-1} g_{i} d \lambda
$$

Observe that one has:

$$
\partial_{t} g(t, x, v)=\frac{1}{2 i \pi} \lim _{\sigma \rightarrow \infty} \int_{\gamma-i \sigma}^{\gamma+i \sigma} \lambda e^{\lambda t}(\lambda I-A)^{-1} g_{i} d \lambda,
$$

and that (3.41) provides a formal solution of the problem (3.39) and (3.40). To complete the proof one has to ensure the convergence of the above integral. Now insert the formula:

$$
\forall g \in D\left(A^{n}\right),(\lambda I-A)^{-1} g=\frac{1}{\lambda} g+\frac{A g}{\lambda^{2}}+\cdots+\frac{A^{n-1} g}{\lambda^{n}}+(\lambda I-A)^{-1} \frac{A^{n}}{\lambda^{n}} g,
$$

with $n=4$ in (3.41) and (3.42). Then,

$$
\begin{aligned}
& \frac{1}{2 i \pi} \lim _{\sigma \rightarrow \infty} \int_{\alpha-i \sigma}^{\alpha+i \sigma} \lambda e^{\lambda t}(\lambda I-A)^{-1} g_{i} d \lambda \\
& =\frac{1}{2 i \pi} \sum_{0 \leq k \leq 4} \lim _{\sigma \rightarrow \infty} \int_{\alpha-i \sigma}^{\alpha+i \sigma} e^{\lambda t} \frac{A^{k} g_{i}}{\lambda^{k}} d \lambda \\
& +\frac{e^{\alpha t}}{2 i \pi} \lim _{\sigma \rightarrow \infty} \int_{\alpha-i \sigma}^{\alpha+i \sigma} e^{i \sigma t}((\alpha+i \sigma) I-A)^{-1} A^{4} g_{i} \frac{d \sigma}{(\alpha+i \sigma)^{4}} .
\end{aligned}
$$

By a residue calculus one has:

$$
\frac{1}{2 i \pi} \lim _{\sigma \rightarrow \infty} \int_{\gamma-i \sigma}^{\gamma+i \sigma} e^{\lambda t} \frac{A^{k-1} g_{i}}{\lambda^{k}} d \lambda=\frac{t^{k-1}}{(k-1) !} A^{k-1} g_{i}, \quad k \geq 1,
$$

while (3.30) gives the estimate

$$
\left\|e^{i \sigma t}((\gamma+i \sigma) I-A)^{-1} A^{4} g_{i} \frac{1}{(\gamma+i \sigma)^{4}}\right\|_{\mathcal{B}} \leq C \frac{(1+|\sigma|)}{|\gamma+i \sigma|^{4}}\left\|A^{4} g_{i}\right\|_{\mathcal{B}}
$$

This implies the strong convergence of the last integral of (3.44). 


\section{The non linear Cauchy Problem}

In Section 3.1, the strong instability of the linearized problem has been proven for any smooth profile $G(v)$ which satisfies the relations

$$
G^{\prime}(0)=0, \quad \int_{-\infty}^{\infty} \frac{G(v)-G(0)}{v^{2}} d v>1 .
$$

Corresponding unstable modes are given by

$$
\phi(t, x, v)=\frac{G^{\prime}(v)}{c^{2}+v^{2}} \int e^{c k t}(v \cos k x-c \sin k x) \hat{\gamma}(k) d k,
$$

for some value of $c$ related to $G$. Their initial values are

$$
g_{\text {in }}(x, v):=\phi(0, x, v)=\frac{G^{\prime}(v)}{c^{2}+v^{2}} \int(v \cos k x-c \sin k x) \hat{\gamma}(k) d k .
$$

As it has been recently observed, instability is a robust property and non linear instability can be systematically deduced from the linearized system. Following the method of Guo and Tice [13], we prove some ill-posedness.

4.1. Weak $\left(H^{m}-H^{1}\right)$ ill-posedness of the nonlinear problem. First recall the definition of a locally $\left(H^{m}-H^{1}\right)$ well-posed Cauchy problem.

Definition 4.1. Let $m \in \mathbf{N}^{*}$. The Cauchy problem

$$
\begin{gathered}
\frac{\partial f}{\partial t}+v \frac{\partial f}{\partial x}=\frac{\partial \rho_{f}}{\partial x} \frac{\partial f}{\partial v}, \quad t>0,(x, v) \in \mathbb{R}^{2}, \\
f(0, x, v)=f_{i}(x, v), \quad(x, v) \in \mathbb{R}^{2},
\end{gathered}
$$

is locally $\left(H^{m}-H^{1}\right)$ well-posed if there is a constant $c_{m}$ such that for any nonnegative initial datum $f_{i} \in H^{m}\left(\mathbb{R}, L^{1}(\mathbb{R})\right)$, there exists a time $T>0$ and a unique nonnegative solution

$$
f \in L^{\infty}\left(0, T ; H^{1}\left(\mathbb{R}, L^{1}(\mathbb{R})\right)\right)
$$

to the problem (4.4)-(4.5), such that

$$
\text { ess } \sup _{t \in(0, T)}\|f(t, \cdot, \cdot)\|_{H^{1}\left(L^{1}\right)} \leq c_{m}\left\|f_{i}\right\|_{H^{1}\left(L^{1}\right)} .
$$

Theorem 4.1. For every $m \in \mathbf{N}^{*}$, the Cauchy problem (4.4)-(4.5) is not locally $\left(H^{m}-H^{1}\right)$ wellposed.

Proof. The proof proceeds by contradiction. Assume the Cauchy problem (4.4)-(4.5) locally $\left(H^{m}-H^{1}\right)$ well-posed. For $f_{i n}=G+\frac{1}{n} g_{i n}$, where $G$ is a function satisfying (4.1) and where $g_{i n}$ is given by (4.3), there is a unique solution $f_{n}=G+g_{n}$ to (4.4)-(4.5). Then $g_{n}$ is the unique solution to the Cauchy problem

$$
\begin{gathered}
\frac{\partial g_{n}}{\partial t}+v \frac{\partial g_{n}}{\partial x}=\frac{\partial \rho_{g_{n}}}{\partial x}\left(G^{\prime}(v)+\frac{\partial g_{n}}{\partial v}\right), \quad t>0,(x, v) \in \mathbb{R}^{2}, \\
g_{n}(0, x, v)=\frac{1}{n} g_{i}(x, v), \quad(x, v) \in \mathbb{R}^{2} .
\end{gathered}
$$

Then $n g_{n}$ is solution to

$$
\begin{gathered}
\frac{\partial\left(n g_{n}\right)}{\partial t}+v \frac{\partial\left(n g_{n}\right)}{\partial x}=\frac{\partial \rho_{n g_{n}}}{\partial x}\left(G^{\prime}(v)+\frac{1}{n} \frac{\partial\left(n g_{n}\right)}{\partial v}\right), \quad t>0,(x, v) \in \mathbb{R}^{2} \\
\left(n g_{n}\right)(0, x, v)=g_{i}(x, v), \quad(x, v) \in \mathbb{R}^{2} .
\end{gathered}
$$

Moreover,

$$
e s s \sup _{t \in(0, T)}\left\|\left(n g_{n}\right)(t, \cdot, \cdot)\right\|_{H^{1}\left(L^{1}\right)} \leq c_{m}\left\|g_{i}\right\|_{H^{1}\left(L^{1}\right)} .
$$


Consequently, $\left(n g_{n}\right)$ converges to some function $\bar{g}$ in the weak star topology of $L^{\infty}\left(w H^{1}(\mathcal{M})\right)$. Given that

$$
\frac{\partial \rho_{n g_{n}}}{\partial x} \frac{\partial\left(n g_{n}\right)}{\partial v}=\frac{\partial}{\partial v}\left(\frac{\partial \rho_{n g_{n}}}{\partial x}\left(n g_{n}\right)\right)
$$

with $\left(\frac{\partial \rho_{n g_{n}}}{\partial x}\right)\left(\operatorname{resp} .\left(n g_{n}\right)\right)$ bounded in $L^{\infty}\left(0, T ; L^{2}(\mathbb{R})\right)\left(\operatorname{resp} . L^{\infty}\left(0, T ; H^{1}\left(\mathbb{R}, L^{1}(\mathbb{R})\right)\right)\right)$,

$$
\frac{1}{n} \frac{\partial \rho_{n g_{n}}}{\partial x} \frac{\partial\left(n g_{n}\right)}{\partial v}
$$

tends to zero in the sense of distributions. And so, $\bar{g}$ is a solution to the linearized equation around $G$ with the initial condition $g_{i n}$. This contradicts (3.26).

\section{Well-Posedness of NON Linear Cauchy PROBlem}

The existence of exponentially growing frequency and the corresponding instability result proven above indicate that stability can only be obtained either in the class of analytic functions or by drastic change of the structure of the problem. Corresponding issues preceding the present article have been already proven. We recall them below to contribute to the understanding of the problem.

5.1. Local in time well-posedness for analytic initial data. As said above the presence of exponential growing modes indicate that well-posedness in full generality may only hold for analytic initial data. This is the object of the article of Jabin and Nouri [15] recalled below. Local in time existence and uniqueness of a solution is proven for appropriate analytic positive initial data, typically of the form

$$
f_{0}(x, v)=\alpha(v) g_{0}(x, v),
$$

where $\int g_{0}(x, v) d v$ is analytic, and $\alpha(v)=\frac{1}{1+|v|^{2}}$ for instance. Its density $\rho$ is obtained as a fixed point of the map $\sigma \mapsto \rho$ defined by the system

$$
\begin{aligned}
& \sigma(t, x) \mapsto g(t, x, v): \partial_{t} g+v \cdot \partial_{x} g-\partial_{x} \sigma\left(\partial_{v} g+\frac{\alpha^{\prime}}{\alpha} g\right)=0, \quad g(0, x, v)=g_{0}(x, v), \\
& \rho(t, x)=\int_{\mathbb{R}_{v}} \alpha(v) g(t, x, v) d v,
\end{aligned}
$$

in the space of functions $g(t, x, v)$ which can be extended analytically in the region

$$
(t, x+i y) \subset\{x \in \mathbb{R}\} \times\left\{|y|<\lambda(t)=\lambda_{0}-K t\right\},
$$

with the norm

$$
\sup _{0<t<\frac{\lambda_{0}}{K}} \sum_{k \in N, l \geq k} \frac{(\lambda(t))^{l-k}}{(k !)^{2}(l-k) !}\left|\partial_{x}^{l} \rho\right|_{\infty}+\int_{0}^{\frac{\lambda_{0}}{K}} \sum_{k \in N, l \geq k} k^{2} \frac{(\lambda(t))^{l-k}}{(k !)^{2}(l-k) !}\left|\partial_{x}^{l} \rho(t, \cdot)\right|_{\infty} d t .
$$

\section{WeLL-POSEDNESS IN THE CLASS OF KINETIC MEASURE SOLUTIONS}

In fact the above instability comes from the freedom of inserting in the initial data perturbations of the form:

$$
\phi(x, v)=\int_{\mathbb{R}_{k}} e^{i k x} \frac{G^{\prime}(v)}{v-\omega^{*}} \hat{\rho}(k) d k,
$$

(resp. in the case of an even profile

$$
\left.\phi(x, v)=\frac{G^{\prime}(v)}{c^{2}+v^{2}} \int(v \cos k x-c \sin k x) \hat{\gamma}(k) d k, \quad \text { with } \hat{\gamma} \text { even }\right)
$$

with $\omega^{*}$ in the general case (resp. $c$ when $G$ is even) solution of the equations:

$$
\Im \omega^{*}>0, \quad \int_{\mathbb{R}_{v}} \frac{G^{\prime}(v)}{v-\omega^{*}}=1
$$

(resp.

$$
\left.\int_{\mathbb{R}_{v}} \frac{G^{\prime}(v) v}{c^{2}+v^{2}} d v=1\right)
$$


Some different type of initial data constrain the problem to live in other settings where the local in time stability is enforced. Below are examples variant of the mono-kinetic solution.

Observe that as long as $V \in W^{2, \infty}\left(\mathbb{R}_{x}\right)$, the Hamiltonian $H=\frac{|v|^{2}}{2}+V(x)$ generates a flow $\Phi_{t}$. Then for any measure $\mu^{i n}$, the push-forward measure

$$
\mu(t)=\Phi_{t} \# \mu^{i n}
$$

belongs to $C_{b}\left(\mathbb{R}_{t}^{+} ; w-\mathcal{M}\left(\mathbb{R}_{x} \times \mathbb{R}_{v}\right)\right)$ and is the unique weak solution of the Liouville equation

$$
\partial_{t} \mu+v \partial_{x} \mu-\partial_{x} V \partial_{v} \mu=0, \quad \mu_{t=0}=\mu^{i n} .
$$

In particular if $\mu^{i n}$ is a bounded positive measure and $V(x)=\rho(x)=\int_{\mathbb{R}_{v}} \mu(x, v, t) d v,(6.2)$ is the Vlasov equation with Dirac potential considered in the present contribution.

For instance, with $\mu^{i n}$ the Kinetic measure given by

$$
\mu^{i n}(x, v)=\rho_{0}(x) \delta_{v-u_{0}(x)},
$$

equation (6.2) is equivalent to the hyperbolic system for isentropic fluid,

$$
\begin{aligned}
& \partial_{t} \rho+\partial_{x}(\rho u)=0, \\
& \partial_{t} u+u \cdot \partial_{x} u+\partial_{x} \rho=0 .
\end{aligned}
$$

The above example has been generalized with the name of water-bag problem ([2], [6], [7]) with further generalization under the name of multi-water-bag equations ([1]). For the water-bag problem, the initial datum is defined by two curves $u_{0}^{-}(x)<u_{0}^{+}(x)$ and the density $\rho_{0}(x, v)=$ $1_{u_{0}^{-}(x)<v<u_{0}^{+}(x)}$. Then the push-forward measure solution of the Liouville equation (6.2) is the measure

$$
\rho(t, x, v)=1_{u^{-}(t, x)<v<u^{+}(t, x)},
$$

with $u^{ \pm}(t, x)$ solutions of the system

$$
\begin{aligned}
& \partial_{t}\left(u^{+}-u^{-}\right)+\partial_{x}\left(\frac{u^{+}+u^{-}}{2}\left(u^{+}-u^{-}\right)\right)=0, \\
& \partial_{t}\left(\frac{u^{+}+u^{-}}{2}\right)+\partial_{x}\left(\frac{u^{+}+u^{-}}{2}\right)^{2}+\partial_{x}\left(u^{+}-u^{-}\right)=0 .
\end{aligned}
$$

Multi-water-bags models are constructed in a similar way.

The important remark in the spirit of the present construction is that (6.4) and (6.5) are well posed problems locally in time but for initial data $\left(\rho_{0}, u_{0}\right)$ or $\left(u_{0}^{-}, u_{0}^{+}\right)$only in $C^{1}$. But these data define in $\mathbb{R}_{x} \times \mathbb{R}_{v}$ measures which are not $(x, v)$ analytic functions. The fact that the problem is well-posed is due to the constraint on the nature of the solutions. No perturbations of different nature are considered.

\section{Conclusion}

This contribution has been devoted to the Cauchy problem for a singular Vlasov equation (VDPE):

$$
\frac{\partial f}{\partial t}+v \frac{\partial f}{\partial x}-\left(\frac{\partial}{\partial x} \int f(t, x, u) d u\right) \frac{\partial f}{\partial v}=0, \quad t>0, \quad(x, v) \in \mathbb{R}_{x} \times \mathbb{R}_{v}
$$

which plays an important role in the analysis of plasma fusion.

The singularity of the mapping

$$
f(t, x, v) \mapsto \partial_{x}\left(\int_{\mathbb{R}_{v}} f(t, x, v) d v\right),
$$

produces a large variety of situations, depending on the nature of the initial profile and the general setting.

Moreover, the VDPE appears also in the $\hbar \rightarrow 0$ limit of the non linear Schrödinger equation, as it has been studied both in [11] and [16]. In these two cases (even if the methods are different), 
the setting is the WKB limit which for the limit case corresponds to the situation of Section 6 , where local in time well-posedness has been set.

Therefore, besides being useful in the modelisation of plasma fusion, the VPDE appears to have a very rich mathematical structure which may be the object of further studies.

Acknowledgements. We wish to thank the hospitality of the Newton Institute for Mathematical Sciences in Cambridge and the Institute for Computational and Experimental Research in Mathematics (ICERM) in Providence, where part of this work was done.

Anne Nouri is partially supported by the ANR GYPSI.

\section{REFERENCES}

[1] Besse, N., Berthelin, F., Brenier, Y., Bertrand, P., 'The multi water-bag model for collisionless kinetic equations', Kinetic and Related Models 2 no. 1, 39-80 (2009)

[2] Besse, N., 'On the waterbag continuum', Arch. Rat. Mech. Anal. 199 no. 2, 453-491 (2011)

[3] Chazarain, J., 'Problèmes de Cauchy abstraits et applications à quelques problèmes mixtes' (French), J. Functional Analysis 7, 386-446 (1971)

[4] Da Prato, G., Mosco, U., 'Semigruppi distribuzioni analitici' (Italian), Ann. Scuola Norm. Sup. Pisa (3) 19 367-396 (1965)

[5] Degond, P., 'Spectral theory of the linearized Vlasov-Poisson equation', Trans. Amer. Math. Soc. 294 no. 2, 435-453 (1986)

[6] DePackh, D.C., 'The Water-bag Model of a Sheet Electron Beam', J. Electron. Control 13, 417-424 (1962)

[7] Feix, M.R., Hohl, F., Staton, L.D., Nonlinear effects in plasmas, in Kalman, G., Feix, M. (eds.) Gordon and Breach, pp 3-21, (1969)

[8] Gérard-Varet, D., Dormy, E., 'On the ill-posedness of the Prandtl equation', J. Amer. Math.Soc. 23 no. 2 , 591-609 (2010)

[9] Gerard-Varet, D., Nguyen, T., 'Remarks on the ill-posedness of the Prandtl equation', to appear in Asymptotic Analysis.

[10] Ghendrih, P., Hauray, M., Nouri, A., 'Derivation of a gyrokinetic model. Existence and uniqueness of specific stationary solutions', Kinetic and Related Models 2 no. 4, 707-725 (2009)

[11] Grenier, E., 'Limite semi-classique de l'équation de Schrödinger non linéaire en temps petit', C. R. Acad. Sci. Paris Sér. I Math. 320 no. 6, 691-694 (1995)

[12] Guo, Y., Nguyen, T., 'A note on Prandtl boundary layers', Comm. Pure. Appl. Math. 64 no. 10, 1416-1438 (2011)

[13] Guo, Y., Tice, I., 'Compressible, inviscid Rayleigh-Taylor instability', to appear in Indiana Univ. Math. J. (2010)

[14] Hauray, M., Nouri, A., 'Well-posedness of a diffusive gyrokinetic model', Annales Institut H.Poincaré 4 no.1, 109-138 (2011)

[15] Jabin, P.-E., Nouri, A., 'Analytic solutions to a strongly nonlinear Vlasov equation', C.R. Acad. Sci. Paris, Sér. 1 349, 541-546 (2011)

[16] Jin, S., Levermore, C. D., McLaughlin, D.W., 'The semiclassical limit of the defocusing NLS hierarchy', Comm. Pure Appl. Math. 52 no. 5, 613-654 (1999)

[17] Krall, N.A., Trivelpiece, A.W., Principles of plasma physics, McGraw Hill (1973)

[18] Lin, Z., Zeng, C., 'Small BGK waves and nonlinear Landau damping', Comm. Math. Phys. 306 no. 2, 291-331 (2011)

[19] Lions, J.-L., 'Les semi groupes distributions' (French), Portugal. Math. 19, 141-164 (1960)

[20] Mouhot, C., Villani, C., 'On Landau damping', Acta Mathematica 207 no. 1, 29-201 (2011)

[21] Penrose, O., 'Electrostatic Instabilites of a Uniform NonMaxwellian Plasma', Phys. Fluids 3 no. 2, 258-265 (1960)

[22] Vlasov, A., Zhur. Eksp. i Teoret. Fiz. 8, 291 (1938)

(C.B.) Université Paris-Diderot, Laboratoire J.-L. Lions, BP187, 4 Place Jussieu, 75252 Paris Cedex 05 France

E-mail address: claude.bardos@gmail.com

(A.N.) Laboratoire D’Analyse, Topologie et Probabilités (UMR 6632), Aix-Marseille Université, 39 rue Joliot-Curie 13453 Marseille Cedex 13 France

E-mail address: anne.nouri@univ-amu.fr 